\title{
Airglow and Aurorae from Dome A, Antarctica
}

\author{
Geoff Sims $^{1}$, Michael C. B. Ashley ${ }^{1}, \mathrm{Xiangqun} \mathrm{Cui}^{2}$, Jon R. Everett ${ }^{1}$, \\ LongLong Feng ${ }^{3,4}$, Xuefei Gong ${ }^{2,4}$, Shane Hengst ${ }^{1}$, Zhongwen $\mathbf{H u}^{2,4}$, \\ Jon S. Lawrence ${ }^{5,6}$, Daniel M. Luong-Van ${ }^{1}$, Anna M. Moore ${ }^{7}$, \\ Reed Riddle $^{7}$, Zhaohui Shang ${ }^{4,8}$, John W. V. Storey ${ }^{1}$, Nick Tothill ${ }^{9}$, \\ Tony Travouillon ${ }^{7}$, Lifan Wang ${ }^{3,4,10}$, Huigen Yang ${ }^{4,11}$, \\ Ji Yang ${ }^{3}$, Xu Zhou ${ }^{4,12}$ and Zhenxi Zhu ${ }^{3,4}$ \\ ${ }^{1}$ School of Physics, University of New South Wales, Sydney NSW 2052, Australia \\ email: g.sims@unsw.edu.au \\ ${ }^{2}$ Nanjing Institude of Astronomical Optics \& Technology, Nanjing 210042, China \\ ${ }^{3}$ Purple Mountain Observatory, Nanjing 210008, China \\ ${ }^{4}$ Chinese Center for Antarctic Astronomy, China \\ ${ }^{5}$ Department of Physics and Astronomy, Macquarie University, Sydney NSW 2109, Australia \\ ${ }^{6}$ Australian Astronomical Observatory, Sydney NSW 1710, Australia \\ ${ }^{7}$ Caltech Optical Observatories, Pasadena, CA, USA \\ ${ }^{8}$ Tianjin Normal University, Tianjin 300074, China \\ ${ }^{9}$ University of Western Sydney, Sydney NSW, Australia \\ ${ }^{10}$ Department of Physics and Astronomy, Texas A\&M University, College Station 77843, USA \\ ${ }^{11}$ Polar Research Institute of China, Shanghai 200136, China \\ ${ }^{12}$ National Astronomical Observatories, Chinese Academy of Science, Beijing 100012, China
}

\begin{abstract}
Despite the absence of artificial light pollution at Antarctic plateau sites such as Dome A, other factors such as airglow, aurorae and extended periods of twilight have the potential to adversely affect optical observations. We present a statistical analysis of the airglow and aurorae at Dome A using spectroscopic data from Nigel, an optical/near-IR spectrometer operating in the 300-850 nm range. The median auroral contribution to the $B, V$ and $R$ photometric bands is found to be $22.9,23.4$ and $23.0 \mathrm{mag} \operatorname{arcsec}^{-2}$ respectively. We are also able to quantify the amount of annual dark time available as a function of wavelength; on average twilight ends when the Sun reaches a zenith distance of $102.6^{\circ}$.
\end{abstract}

Keywords. Dome A, site testing, airglow, aurora, sky brightness

\section{Airglow and Aurorae}

Using the Nigel spectrometer (Sims et al. 2010), we investigated the sky brightness contribution from the dominant emission lines in the optical photometric bands: $B$ (391.4 and $427.8 \mathrm{~nm}) ; V(557.7 \mathrm{~nm})$; and $R(630.0$ and $636.4 \mathrm{~nm})$. A sample histogram for the $V$ band is shown in Figure 1.

\section{Twilight}

At high latitudes the total amount of useable dark time is critically dependent on the solar zenith distance at which it becomes dark. One interesting consequences of the low aerosol content of the Antarctic atmosphere is that it results in the sky darkening 


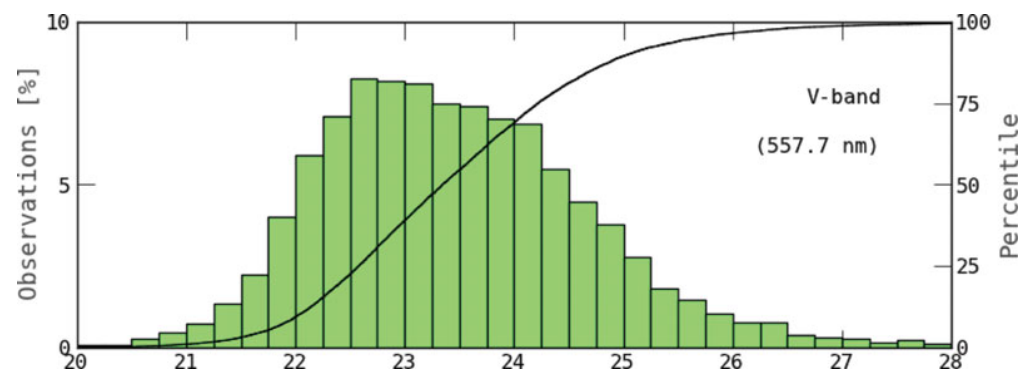

Figure 1. Probability histogram showing the contribution of the $557.7 \mathrm{~nm}$ line to sky brightness in the $V$ band.

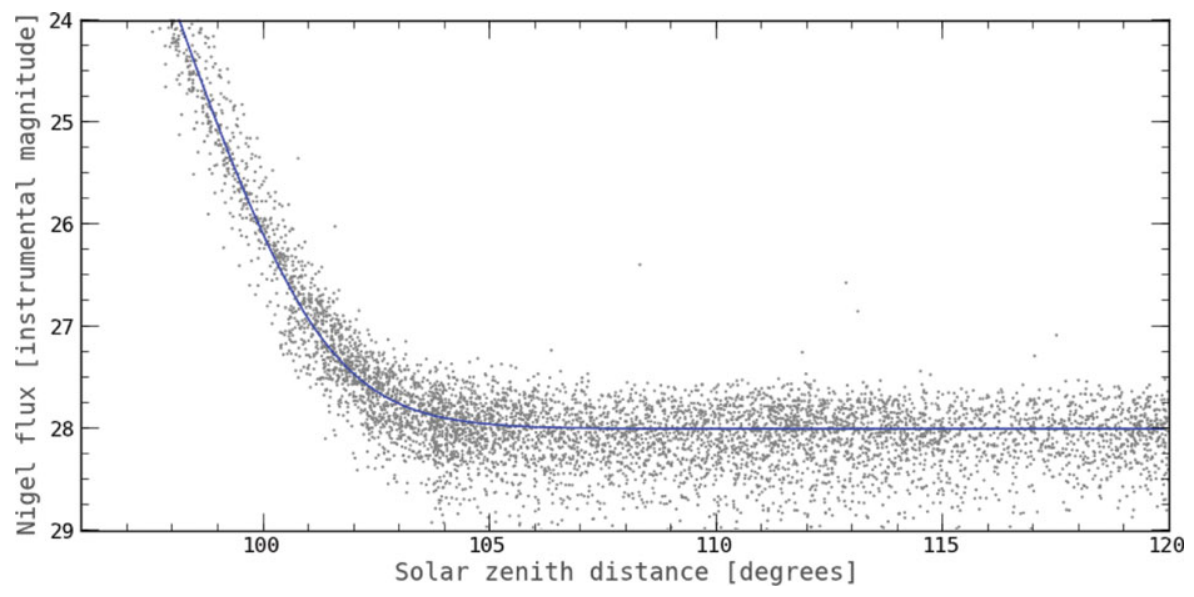

Figure 2. Determining the solar zenith distance at which twilight ends.

faster than at temperate latitudes. Figure 2 shows data from one wavelength interval, demonstrating that twilight effectively ends at a solar zenith distance of $\sim 103^{\circ}$.

\section{Future Work}

Auroral statistics for 2009 from Nigel have recently been published (Sims et al. 2012). Our data also complement photometric images from the Gattini camera (Moore et al. 2010) which uses $B, V$ and $R$ filters. This will allow the background sky brightness at Dome A to be disentangled from the various airglow and auroral emission lines (Moore et al., in prep.).

\section{Acknowledgements}

The author acknowledges the Astronomical Society of Australia (ASA) for providing travel support to attend this meeting.

\section{References}

Moore, A. et al. 2010, Proc. SPIE, 7733, 77331S

Sims, G. et al. 2010, Proc. SPIE, 7733, 77334M

Sims, G. et al. 2012, Publ. Astron. Soc. Pac., 124, 637 